\title{
E37 EVALUATION OF THE PERFORMANCE OF COMPLEX WELLS BY BOUNDARY INTEGRAL METHODS
}

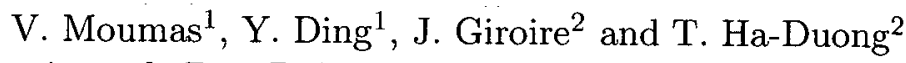 \\ ${ }^{1}$ Institut Français du Pétrole, 1 et 4 av. de Bois Préau, 92852 Rueil Malmaison, France \\ ${ }^{2}$ Université de Technologie de Compiègne, 60200 Compiègne, France
}

\begin{abstract}
Classical numerical methods like finite differences, finite volumes or finite elements do not provide sufficientl precise results around the well. This is a consequence of the scale difference between the size of the reservo (several kilometers) and the radius of the well (several centimeters). Hybrid meshes with grid refinement aroun the well can improve the precision, but they are difficult to apply for modelling complex wells. To overcome th scale problem between the reservoir size and the wellbore radius, boundary integral methods can be used.

In this paper, a stratified reservoir is considered with an arbitrary geometry. The permeability is suppose homogeneous in each reservoir layer. The performance of complex wells is evaluated in such a reservoir b. boundary integral methods. The boundary integral equation is developped on the reservoir boundary, on th interfaces between the different layers as well as on the wellbore boundary. Since we are only interested in th performance of the well, we can restrict the problem to a stationary one.

The discretization of the integral equations is performed by a Galerkin method. The integral equation on th well can be reduced to a $1 \mathrm{D}$ equation. Some numerical examples will be presented to show the efficiency of th method.
\end{abstract}

\section{Introduction}

Horizontal and multi-lateral wells are more and more used in petroleum engineering to improve oi recovery. Modelling of well production is a very important issue for petroleum engineers. A numerica model for the evaluation of well performances can allow them to optimize the productivity of the reservoir according to the architecture or the location of the complex wells.

In this paper, evaluation of well performances will be treated by boundary integral methods, wellknown in domains like acoustics, electromagnetism, or mechanics (see [2]), but recently applied ir petroleum engineering. Until now, applications of boundary integral methods in petroleum engineering were generally limited in $2 \mathrm{D}$, and the wells were modelled using sink / source terms. In this paper, we will extend the application of boundary integral methods in 3D for a stratified reservoin with arbitrary reservoir geometry.

The steady-state flow problem is considered for flow modelling. The well is not modelled using a line sink / source, and the real well geometry is considered.

Boundary integral equations are established on the reservoir outer boundaries, at the interfaces between the layers, and on the well boundary. A Galerkin approach is used to solve the boundary integra equations. Numerical integration on the well boundary can be reduced to a $1 \mathrm{D}$ problem.

Numerical results show the efficiency of our method.

\section{Mathematical model}

In this paper, we consider a 3D stratified reservoir with an arbitrary geometry, a well is placed in the reservoir. Let $\Omega$ denote the petroleum reservoir which does not include the domain occupied by the well. The reservoir is divided into homogeneous layers $\Omega_{i}, i=1, \ldots, N_{R}$, with boundary $\Gamma_{i}$. An integral equation is established on the reservoir boundary $\Gamma$ which is composed by the outer boundaries $\Gamma_{D} \cup \Gamma_{N}$, by the well boundary $\Gamma_{w}$, and by the interfaces between the layers $\Gamma_{i j}=\Gamma_{i} \cap \Gamma_{j}$.

To simplify, we first present the model for a homogeneous reservoir (see figure (a)), and then we will generalize the model to a stratified medium. 


\subsection{Homogeneous reservoir}

Let $x=\left(x_{1}, x_{2}, x_{3}\right)$ denote a point in a Cartesian coordinate system and $t$ denote time in the interval $[0, T]$.

If gravity is neglected, the single-phase flow is governed by a diffusion equation:

$$
\left.\left.\operatorname{div}(\overline{\bar{K}} . \nabla p(x, t))=\left(\phi \mu c_{f}\right) \frac{\partial p(x, t)}{\partial t} \quad x \in \Omega, t \in\right] 0, T\right]
$$

where $p$ is the pressure of the fluid in the porous medium, the tensor $\overline{\bar{K}}$ stands for the reservoir permeability, and $\phi$ and $\mu$ refer respectively to the porosity of the medium and the viscosity of the fluid. Initial condition is given by: $p(x, 0)=p_{0}, x \in \Omega$.

The diffusion equation (1) is usually used for modelling well-testing. Here we are only interested in the performance of the well, so that we proceed with the study of the stationary problem:

$$
\operatorname{div}\left(\frac{\overline{\bar{K}}}{\mu} \cdot \nabla p(x)\right)=0 \quad x \in \Omega .
$$

We suppose the tensor $\overline{\bar{K}}$ diagonal with diagonal elements $\left(k_{1}, k_{2}, k_{3}\right)$.

Unlike in the work in the literature (Koh and Tiab [8], Pecher and Stanislav [12], Mohamad and Numbere [10], or Oguztoreli and Wong [13]), equation (2) does not include any sink or source term. The contribution of the well is incorporated to the problem by means of the following boundary condition:

$$
Q=\frac{\tilde{k}}{\mu} \int_{\Gamma_{w}} \frac{\partial p(x)}{\partial(\overline{\bar{K}} n)} \mathrm{d} \gamma(x)
$$

where $Q$ is the prescribed total flow rate, $n$ is the outward normal vector to $\Gamma_{w}$,

and $\frac{\partial p}{\partial(\overline{\bar{K}} n)}=\frac{1}{\tilde{k}} \overline{\bar{K}} \nabla p \cdot n$, where $\tilde{k}=\left(k_{1} k_{2} k_{3}\right)^{1 / 3}$.

Now let us write the boundary conditions.

\subsubsection{Reservoir outer boundary conditions}

At the outer boundary, two types of conditions are considered:

$$
\begin{aligned}
p(x) & =p_{D}(x) & & x \in \Gamma_{D} \\
\frac{\partial p(x)}{\partial(\overline{\bar{K}} n)} & =g(x) & & x \in \Gamma_{N}
\end{aligned}
$$

The Dirichlet condition on $\Gamma_{D}$ (constant pressure) corresponds for instance to the presence of a large capacity aquifer.

The Neumann condition imposes a fluid flow; this happens for example when we have an impermeable barrier: the flux is therefore null $\left(g \equiv 0\right.$ on $\left.\Gamma_{N}\right)$.

\subsubsection{Well boundary conditions}

For simplicity of notation, we consider a single horizontal well with radius $r_{w}$ and length $L$.

We can have two different conditions on $\Gamma_{w}$ : infinite conductivity condition or finite conductivity condition, according to whether we take into account the effects of pressure drop or not.

Assuming the pressure $p$ constant in each cross-section of the well, we can write $p$ as a function of the axis variable $l \in[0, L]$ along the well. 
Infinite conductivity If we do not take into account the effects of pressure drop, we assume th pressure constant along the well:

$$
p(l)=p_{w}, \quad l \in[0, L]
$$

This is known as infinite conductivity condition in petroleum engineering, and is widely accepted.

Finite conductivity If we take into account the effects of pressure drop, we have a finite conduc tivity condition. The corresponding equation can be written in the following form:

$$
\begin{aligned}
\frac{\partial p(l)}{\partial l} & =C_{w} q_{w}^{2}(l), & & l \in[0, L] \\
\text { with } & q_{w}(l) & =\int_{\Gamma_{w, l}} \frac{\partial p(x)}{\partial(\overline{\bar{K}} n)} \mathrm{d} \gamma(x), & l \in[0, L],
\end{aligned}
$$

where $C_{w}$ is a constant, and $n$ is the outward normal vector to $\Gamma_{w, l}$.

$\Gamma_{w, l}$ denotes the part of the well boundary $\Gamma_{w}$ from which the flow comes at the section $S_{l}$, as showr here for a complex well in figure (b).

The infinite conductivity condition (6) is a particular case of the finite conductivity condition (take $\left.C_{w}=0\right)$.

Equations $(2-5)$ and $(7-8)$ constitute the governing equations of the model with finite conductivity condition in the case of a homogeneous reservoir.

In the next section, we give additional conditions to generalize this model to a stratified reservoir.

\subsection{Stratified reservoir}

To obtain the model for a stratified reservoir, we begin by writing the previous model (2-5) and (78 ) indexed by $i$ for each layer $\Omega_{i}$ (which is supposed homogeneous as seen previously) and then we complete the model with the following interface conditions.

Pressure and flux continuity conditions must be satisfied at the zonal interfaces $\Gamma_{i j}$ :

$$
\begin{aligned}
p_{i} & =p_{j} & & \text { on } \Gamma_{i j} \\
\tilde{k}_{i} \frac{\partial p_{i}}{\partial\left(\overline{\bar{K}}_{i} n_{i}\right)} & =-\tilde{k}_{j} \frac{\partial p_{j}}{\partial\left(\overline{\bar{K}}_{j} n_{j}\right)} & & \text { on } \Gamma_{i j}
\end{aligned}
$$

\section{Integral methods}

Two types of methods can be used to solve this problem: volumetric numerical methods (such as control-volume method) or boundary integral methods. Numerical methods can solve complicated problems, for example heterogeneous media or arbitrary geometry reservoirs. However, numerical methods suffer from a lack of precision around the well: it comes from an important scale difference between the size of the reservoir (several kilometers) and the radius of the well $(\leq 0.15 \mathrm{~cm})$. Hybrid meshes with grid refinement around the well can improve the precision, but they are difficult to apply for complex wells. To overcome the scale problem between the reservoir size and the wellbore radius, boundary integral methods can be used.

Boundary integral methods are widely used in electromagnetism, mechanics [2]... but their applications in petroleum engineering are somewhat rare. Liggett and Liu [9] in 1979 were the first to use such methods in petroleum engineering, then some examples are the work by Koh and Tiab [8], Pecher and Stanislav [12], Kikani and Horne [7], Oguztoreli and Wong [13], and more recently Ding [4] and [5] for complex well modelling.

The interest of such methods is that the problem can be formulated on the boundaries: the equations are written on the reservoir outer boundaries, on the well boundary, and on the interfaces between the layers. We need to mesh only the boundaries, and no more all the reservoir. 
In the next section, we present the integral formulation of our problem and we will focus on the linear model (infinite conductivity condition on the well) for a homogeneous reservoir.

Finite conductivity condition, corresponding to a non-linear model, will be treated in future work by a Newton method.

\section{Integral representation of the model}

Two kinds of integral representations can be written as (see Bonnet [2]):

$$
c p=-V q+S p, \quad \text { on } \Gamma,
$$

or:

$$
c q=-H q+D p \quad \text { on } \Gamma
$$

where $q$ stands for the normal derivative of the pressure $\frac{\partial p}{\partial\left(\overline{\bar{K}} n_{x}\right)}, c$ is a constant depending on the boundary $\Gamma$ (if $\Gamma$ is regular, $c=1 / 2$, if not see [12]), and the operators $V, S, H, D$ are defined by :

$$
\begin{array}{rlrl}
V q & =\int_{\Gamma} G(x-y) q(y) \mathrm{d} \gamma(y) & S p=\int_{\Gamma} \frac{\partial G(x-y)}{\partial\left(\overline{\bar{K}} n_{y}\right)} p(y) \mathrm{d} \gamma(y) \\
D p=\frac{\partial}{\partial n_{x}} \int_{\Gamma} \frac{\partial G(x-y)}{\partial\left(\overline{\bar{K}} n_{y}\right)} p(y) \mathrm{d} \gamma(y) & H q=\int_{\Gamma} \frac{\partial G(x-y)}{\partial\left(\overline{\bar{K}} n_{x}\right)} q(y) \mathrm{d} \gamma(y)
\end{array}
$$

$G$ is the Green function associated to the problem and is given by:

$$
G(x-y)=\frac{1}{4 \pi} \frac{1}{\left(a^{2}\left(x_{1}-y_{1}\right)^{2}+b^{2}\left(x_{2}-y_{2}\right)^{2}+c^{2}\left(x_{3}-y_{3}\right)^{2}\right)^{1 / 2}}
$$

where $a=\frac{\left(k_{1} k_{2} k_{3}\right)^{1 / 6}}{k_{1}^{1 / 2}}, b=\frac{\left(k_{1} k_{2} k_{3}\right)^{1 / 6}}{k_{2}^{1 / 2}}, c=\frac{\left(k_{1} k_{2} k_{3}\right)^{1 / 6}}{k_{3}^{1 / 2}}$.

We have to choose a formulation for each boundary. In petroleum engineering, boundary integral equations correspond to the first formulation (9). The second formulation (10) has not been used yet in petroleum engineering whereas its application in acoustics or mechanics has been successful. The interest of $(10)$ is that this formulation is more stable than the first one (9) for Dirichlet boundary conditions (see [11] or first kind integral equations / second kind integral equations in Bonnet [2]).

On the Dirichlet boundary $\Gamma_{D}$, we have written the second representation (10), and on the Neumann boundary $\Gamma_{N}$ as well as on the well boundary $\Gamma_{w}$, the first formulation (9):

$$
\begin{array}{ll}
c q=-H q+D p & \text { on } \Gamma_{D} \\
c p=-V q+S p & \text { on } \Gamma_{N} \\
c p=-V q+S p & \text { on } \Gamma_{w}
\end{array}
$$

Our results will show the efficiency of this representation.

\section{Numerical aspects}

To solve the boundary integral equations, a Galerkin method has been performed.

We choose as basis functions $\left(\psi_{i}^{h}\right)_{i=1, \ldots, N}$, the finite element functions $\mathbb{P}^{1}, N$ is the total number of nodes in the mesh. We denote by $p^{h}$ and $q^{h}$ the approximations of the unknowns $p$ and $q$, and we decompose $p^{h}$ and $q^{h}$ in the basis $\left(\psi_{i}^{h}\right)_{i=1, \ldots, N}$ :

$$
p^{h}(x)=\sum_{j=1}^{N} p_{j} \psi_{j}^{h}(x), \quad q^{h}(x)=\sum_{j=1}^{N} q_{j} \psi_{j}^{h}(x) .
$$


Let $\Gamma_{h}$ be an approximation of the boundary $\Gamma$. After multiplying the three previous integral equation by a basis function $\psi_{i}^{h}$ and then integrated once more over the boundary $\Gamma_{h}$, it is necessary to evaluate the five following terms:

$$
\begin{aligned}
& \int_{\Gamma_{h}} \psi_{i}^{h}(x) \psi_{j}^{h}(x) \mathrm{d} \gamma_{h}(x) \\
& \int_{\Gamma_{h}} \int_{\Gamma_{h}} \psi_{i}^{h}(x) \psi_{j}^{h}(y) G(x-y) \mathrm{d} \gamma_{h}(y) \mathrm{d} \gamma_{h}(x) \\
& \int_{\Gamma_{h}} \int_{\Gamma_{h}} \psi_{i}^{h}(x) \psi_{j}^{h}(y) \frac{\partial G(x-y)}{\partial\left(\overline{\bar{K}} n_{y}\right)} \mathrm{d} \gamma_{h}(y) \mathrm{d} \gamma_{h}(x) \\
& \int_{\Gamma_{h}} \int_{\Gamma_{h}} \psi_{i}^{h}(x) \psi_{j}^{h}(y) \frac{\partial G(x-y)}{\partial\left(\overline{\bar{K}} n_{x}\right)} \mathrm{d} \gamma_{h}(y) \mathrm{d} \gamma_{h}(x) \\
& \int_{\Gamma_{h}} \psi_{i}^{h}(x) \frac{\partial}{\partial\left(\overline{\bar{K}} n_{x}\right)} \int_{\Gamma_{h}} \psi_{j}^{h}(y) \frac{\partial G(x-y)}{\partial\left(\overline{\bar{K}} n_{y}\right)} \mathrm{d} \gamma_{h}(y) \mathrm{d} \gamma_{h}(x)
\end{aligned}
$$

The first integral (14) comes from the left-hand side of equations (11),(12), (13) and is very easy to calculate, the second integral (15) comes from the operator $V$ appearing in (12) and (13), the third (16) from the operator $S$ in (12) and (13), the fourth (17) from the operator $H$ in (11), and the fifth (18) from the operator $D$ in (11).

\subsection{Singularities}

Integrals (15), (16) and (17) are weakly singular integrals: the kernels $G$ and $\partial G / \partial(\overline{\bar{K}} n)\left(n=n_{x}\right.$ or $n_{y}$ ) present singularities at $x \rightarrow y$. The kernels $G$ and $\partial G / \partial(\overline{\bar{K}} n)$ behave as $r^{-1}$, where $r=|x-y|$. By taking into account these singularities, we can obtain an analytic formula for the integral with respect to $y$ and then the integral with respect to $x$ is performed by numerical integration. On the wellbore boundary, an analytic formula can be obtained for both $x$ and $y$ integrals along the well (see section (5.2)).

The difficult point here is integral (18). This is a hypersingular integral: the trouble is that the normal derivative $\partial / \partial\left(\overline{\bar{K}} n_{x}\right)$ cannot be included into the integral with respect to $y$, otherwise this integral would not be defined. By same techniques as in Giroire [6], we can obtain the following formula:

$$
\begin{aligned}
& \int_{\Gamma_{h}} \psi_{i}^{h}(x) \frac{\partial}{\partial\left(\overline{\bar{K}} n_{x}\right)} \int_{\Gamma_{h}} \psi_{j}^{h}(y) \frac{\partial G(x-y)}{\partial\left(\overline{\bar{K}} n_{y}\right)} \mathrm{d} \gamma_{h}(y) \mathrm{d} \gamma_{h}(x) \\
= & -\frac{1}{4 \pi} \int_{\Gamma_{h}} \int_{\Gamma_{h}} \frac{\text { rot }_{\Gamma_{h}} \psi_{i}^{h}(x) \cdot \text { rot }_{\Gamma_{h}} \psi_{j}^{h}(y) \mathrm{d} \gamma_{h}(y) \mathrm{d} \gamma_{h}(x)}{\left(a^{2}\left(x_{1}-y_{1}\right)^{2}+b^{2}\left(x_{2}-y_{2}\right)^{2}+c^{2}\left(x_{3}-y_{3}\right)^{2}\right)^{1 / 2}}
\end{aligned}
$$

where $\tilde{\operatorname{rot}}_{\Gamma_{h}} u=\left(\frac{1}{\tilde{k}^{1 / 2}} \overline{\bar{K}}^{1 / 2} \nabla u\right) \wedge\left(\frac{1}{\tilde{k}^{1 / 2}} \overline{\bar{K}}^{1 / 2} n\right), \overline{\bar{K}}^{1 / 2}$ is the diagonal matrix with diagonal elements $\left(k_{1}^{1 / 2}, k_{2}^{1 / 2}, k_{3}^{1 / 2}\right), \wedge$ is the vectorial product, and $n$ is the outward normal to the boundary $\Gamma_{h}$.

The basic idea to obtain this result is to transform the normal derivatives $\partial / \partial\left(\overline{\bar{K}} n_{x}\right)$ and $\partial / \partial\left(\overline{\bar{K}} n_{y}\right)$ into tangential derivatives on the basis functions $\psi_{i}^{h}$ and $\psi_{j}^{h}$ (for more details see [11]).

Now it is worth pointing out that this formula is very simplified: since the basis functions $\psi_{i}^{h}$, 
$i=1, \ldots, N$, are polynomials of degree 1 , the terms $\tilde{\operatorname{rot}}_{\Gamma_{h}} \psi_{i}^{h}(x)$ and $\tilde{\operatorname{rot}}_{\Gamma_{h}} \psi_{j}^{h}(y)$ become constants. Then the crucial advantage of using a Galerkin method lies in the fact that the hypersingularity can be treated easily thanks to the additionnal integration and the basis function.

For boundary discretization, $\Gamma_{D}^{h}$ and $\Gamma_{N}^{h}$ are discretized by triangles.

The well boundary $\Gamma_{w}^{h}$ is discretized by cylinder segments. As mentioned in section 3 , the radius $r_{w}$ of the well is very small with respect to the size of the reservoir, and then with respect to the length $L$ of the well. Under this condition, integration along a well cylindrical segment can be reduced to a line integration, this point will be explained in the next section.

\subsection{Special treatment for the well}

Our aim is to evaluate a 2D integral (on a cylinder) by a 1D integral (on a line). The difficulty is that the normal derivative is not defined in $1 \mathrm{D}$.

We recall that we have supposed that the unknowns $p$ and its normal derivative $q$ are constant over a well boundary cross-section.

Let $h_{i}$ be the discretized well segment length. If $h_{i}$ is sufficiently large with respect to the radius $r_{w}$, we can calculate the integral terms analytically.

For simplicity of notation, we consider a horizontal well in an isotropic medium. We will take for example the integral (16) and we will restrict ourselves to the treatment of the part of this integral on $\Gamma_{w}^{h} \times \Gamma_{w}^{h}$. When discretized, this integral is expressed as:

$$
\begin{aligned}
& A_{i j}=2 r_{w} \int_{0}^{L} \psi_{i}^{h}\left(l^{\prime}\right) \mathrm{d} l^{\prime} \int_{0}^{L} \psi_{j}^{h}(l) \mathrm{d} l \int_{0}^{\pi / 2} \frac{2 r_{w}^{2} \sin ^{2} \theta \mathrm{d} \theta}{\left[4 r_{w}^{2} \sin ^{2} \theta+\left(l-l^{\prime}\right)^{2}\right]^{3 / 2}} \\
& A_{i j}=2 r_{w} \sum_{k=1}^{N-1} \int_{l_{k}}^{l_{k+1}} \psi_{i}^{h}\left(l^{\prime}\right) \mathrm{d} l^{\prime} \sum_{m=1}^{N-1} \int_{l_{m}}^{l_{m+1}} \psi_{j}^{h}(l) \mathrm{d} l \int_{0}^{\pi / 2} \frac{2 r_{w}^{2} \sin ^{2} \theta \mathrm{d} \theta}{\left[4 r_{w}^{2} \sin ^{2} \theta+\left(l-l^{\prime}\right)^{2}\right]^{3 / 2}}
\end{aligned}
$$

If the distance between elements $\left[l_{k}, l_{k+1}\right]$ and $\left[l_{m}, l_{m+1}\right]$ is sufficiently large, we can neglect the denominator term $4 r_{w}^{2} \sin ^{2} \theta$ with respect to $\left(l-l^{\prime}\right)^{2}$, and then the integral has an analytic formulation. If not, we can first integrate analytically with respect to $l$ and $l^{\prime}$. By some variable transformations, we can write that:

$A_{i j}=2 r_{w} \sum_{k \in \mathcal{I}} \sum_{m \in \mathcal{J}} I_{i k j m}$, where $\mathcal{I}=\{i-1, i\}$ if $2 \leq k \leq N-2, \mathcal{I}=\{i\}$ if $k \in\{1, N-1\}$

and $I_{i k j m}$ is defined by:

$$
\mathcal{J}=\{j-1, j\} \text { if } 2 \leq m \leq N-2, \mathcal{J}=\{j\} \text { if } m \in\{1, N-1\}
$$

$$
I_{i k j m}=\frac{1}{\left|b_{i k} b_{j m}\right|} \int_{0}^{\pi / 2} \int_{0}^{b_{i k}} \int_{0}^{b_{j m}} \frac{2 r_{w}^{2} \sin ^{2} \theta s s^{\prime} \mathrm{d} s \mathrm{~d} s^{\prime} \mathrm{d} \theta}{\left[4 r_{w}^{2} \sin ^{2} \theta+\left(s-s^{\prime}+c_{i k j m}\right)^{2}\right]^{3 / 2}}
$$

where $b_{i k}=l_{i}-\left(\delta_{i k} l_{k+1}+\delta_{i k+1} l_{k}\right), b_{j m}=l_{j}-\left(\delta_{j m} l_{m+1}+\delta_{j m+1} l_{m}\right)$,

$c_{i k j m}=\left(\delta_{j m} l_{m+1}+\delta_{j m+1} l_{m}\right)-\left(\delta_{i k} l_{k+1}+\delta_{i k+1} l_{k}\right)$, and $\delta$ is the Dirac function.

After integrating with respect to $s$ and $s^{\prime}$, we can obtain:

$I_{i k j m}=\frac{1}{\left|b_{i k} b_{j m}\right|} \int_{0}^{\pi / 2}\left\{f\left(\theta, b_{j m}-b_{i k}+c_{i k j m}\right)-f\left(\theta, b_{j m}+c_{i k j m}\right)-f\left(\theta,-b_{i k}+c_{i k j m}\right)+f\left(\theta, c_{i k j m}\right)\right\} \mathrm{d} \theta$

where

$f(\theta, t)=c_{i k j m} r_{w}^{2} \sin ^{2} \theta \ln \left(t+\sqrt{4 r_{w}^{2} \sin ^{2} \theta+t^{2}}\right)-\frac{1}{6}\left(4 r_{w}^{2} \sin ^{2} \theta+t^{2}\right)^{3 / 2}+\lambda(t)\left(4 r_{w}^{2} \sin ^{2} \theta+t^{2}\right)^{1 / 2}$,

and $\lambda(t)$ is a constant depending on $t$ and on $b_{i k}, b_{j m}, c_{i k j m}$ (for more details see [11]).

We can notice that $t$ is depending on $h_{i}$ and $h_{j}$. We can show that if $h_{i}, h_{j}$ are sufficiently large with respect to $r_{w}$, we have $|t|>>r_{w}$ or $t=0$.

Then we can neglect $4 r_{w}^{2} \sin ^{2} \theta$ in the last two terms if $t \neq 0$. For the first term, if $t>0$, we can also neglect $4 r_{w}^{2} \sin ^{2} \theta$ inside the logarithm, which is then reduced to $\ln (2|t|)$; if $t<0$, we multiply the terms 
inside the logarithm by their conjugate expression, and then we can make the same simplificatior Thanks to this method, we can obtain an analytic formula.

If $t=0$, standard integration gives an analytic formula.

With this method, we have seen that we can obtain an analytic formula for all matrix elements.

\section{First results and validations}

The first validation test is a horizontal well in an infinite reservoir. We assume the pressure to be at infinity. An infinite conductivity condition is taken. Then the integral representation of the mode consists of equation (13).

The well has length $L=1000 \mathrm{~m}$, and radius $r_{w}=0.108 \mathrm{~m}$. The pressure along the well is $p_{w}=1$ bar The reservoir is isotropic with permeability $1 \mathrm{mD}$.

The well is discretized into $N=8$ non uniform segments. We have taken as reference solution solution computed by an integral method discretized with a collocation method (see [2]). Figure (c shows the flux along the well axis.

Figure (d) shows the convergence of the Galerkin method, the well is successively discretized int $N=8$ segments, $N=10$ segments, $N=20$ segments and $N=50$ segments. Here the mesh uniform.

These two results show that the formulation chosen on the well is a good formulation.

\section{Conclusion}

Boundary integral methods have been applied to treat a 3D steady-state problem for advanced wel modelling in petroleum engineering. The boundary integral equations have been discretized with Galerkin method, and the integrals on the well have been simplified to model the well as a line by nonusual method. The preliminary tests give promising results.

Future work will be to extend these results to a stratified reservoir with arbitrary geometry, with finite conductivity condition. Then thanks to Laplace transform, this will be generalized to the transient problem.

\section{References}

[1] K. Aziz and A. Settari - Petroleum reservoir simulation, Elsevier Applied Science Publishers, London, 1979.

[2] M. Bonnet - Equations intégrales et éléments de frontières, Applications en mécanique des solides et des fluides, CNRS Editions / Eyrolles, 1995.

[3] G. Chavent and J. Jaffré, Mathematical models and finite elements for reservoir simulation, Studies in Mathematics and its applications, Elsevier Science Publishers, 17, 1986.

[4] Y. Ding - Transient Pressure Solution in the presence of pressure drop in the wellbore, SPE, 56614, 1999 .

[5] L. Farina, Y. Ding and T. Ha-Duong - Transient pressure solution for a horizontal well in a petroleum reservoir by boundary integral methods, International Journal for Numerical Methods in Engineering, 49, pp. $669-679,2000$.

[6] J. Giroire - Cours de DEA de l'Université de Nantes, 1992.

[7] J. Kikani and R.N. Horne - Pressure-Transient Analysis of Arbitrarily Shaped Reservoirs With the Boundary-Element Method, SPE, 18159, pp. 53-60, 1998.

[8] L.S. Koh and D. Tiab - A Boundary-Element Algorithm for Modeling 3D Horizontal Well Problems Using 2D Grids, SPE, 26228, pp. 91-106, 1993. 
[9] J.A. Liggett and P.L.F. Liu - Unsteady Flow in Confined Aquifers : A Comparison of Two Boundary Integral Methods, Water Resources Research, 15:4, pp.861-866, 1979.

[10] M.N. Mohamad-Ibrahim and D.T. Numbere - Boundary element analysis of the productivity of complex petroleum well configurations, Boundary Element Technology XIII, WITpress, pp. 25-34, 1999.

[11] V. Moumas - Modélisation de l'écoulement monophasique pour les puits complexes dans un réservoir stratifié à géométrie quelconque, $\mathrm{PhD}$ thesis, in preparation.

[12] R. Pecher and J.F. Stanislav - Boundary Element techniques in petroleum reservoir simulation, Journal of Petroleum Engineering, 17, pp.353-366, 1997.

[13] M. Oguztoreli and D.W. Wong - VERTEX : A New Modeling Method used to Direct Field Development, $S P E$, 39806, pp. 467-476, 1998.

\section{Figures}

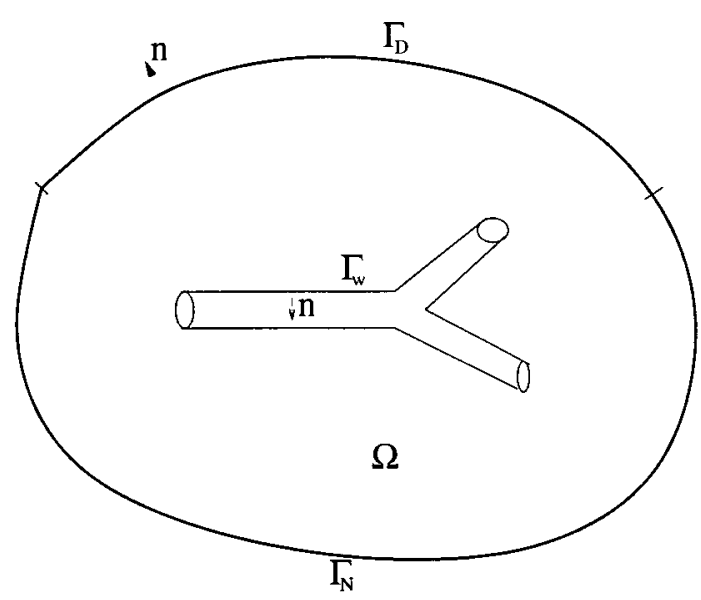

(a) Homogeneous reservoir

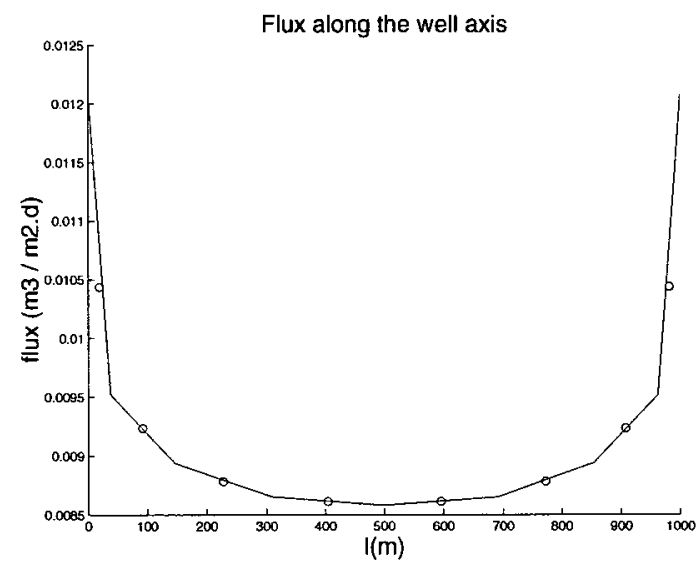

(c) -: Galerkin method o: Collocation method

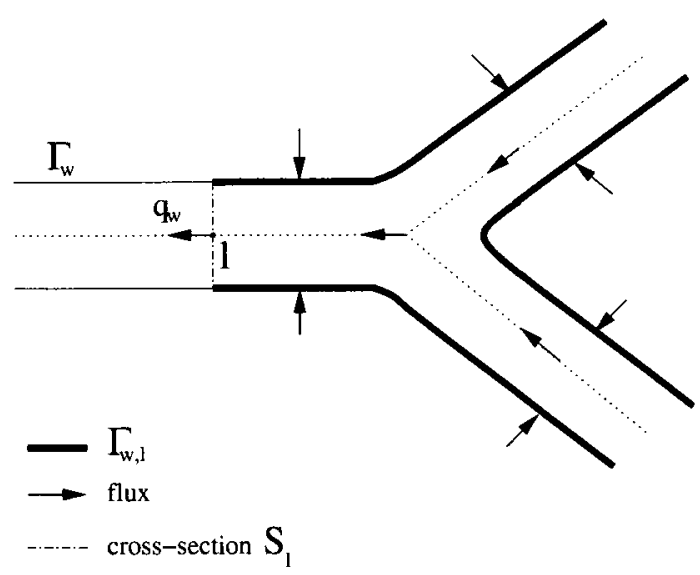

(b) Part $\Gamma_{w, l}$ of the well boundary $\Gamma_{w}$

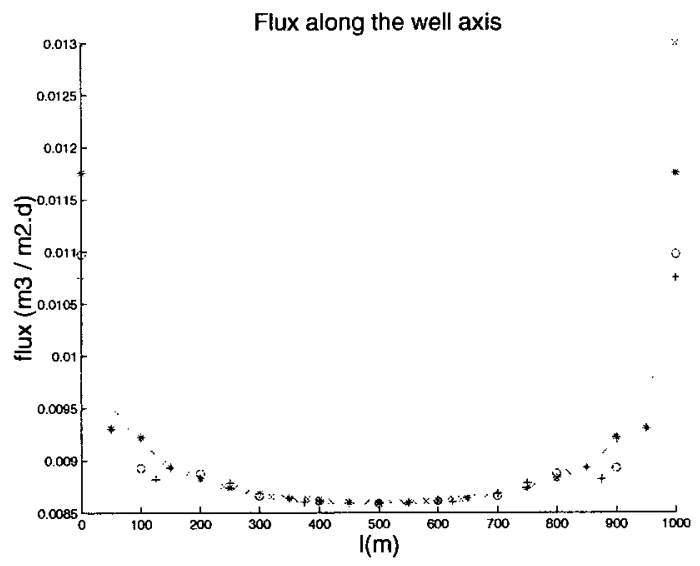

(d) $+: \mathrm{N}=8$ o: $\mathrm{N}=10 *: \mathrm{N}=20 \mathrm{x}: \mathrm{N}=50$ 